\title{
Identification and characterization of microglia/macrophages in the granuloma microenvironment of encephalic schistosomiasis japonicum
}

\author{
Zhoubin Tan ${ }^{1}$, Zhuowei Lei ${ }^{2}$, Zhuo Zhang ${ }^{1,3}$, Huaqiu Zhang ${ }^{1}$, Kai Shu' ${ }^{1}$ Feng Hu ${ }^{1 *}$ and Ting Lei ${ }^{1 *}$
}

\begin{abstract}
Background: Egg-induced immune response and granuloma formation are thought to be the basis of central nervous system (CNS)-related clinical symptoms of Schistosoma japonicum. Microglia/macrophages are the major immune cells involved in detection and subsequent elimination of pathogens and injured tissue in the brain. However, little is known about their role in the pathogenesis of neuroschistosomiasis. The main purpose of the study is to clarify the pathological involvement of microglia/macrophages in the pathogenesis of neuroschistosomiasis (NS).

Methods: Staining techniques were applied to the granuloma tissues excised from 4 patients, as well as mice model which was established by microinjecting viable S. japonicum eggs into the brain. Clinical features of the patients and neurological symptoms in mice were also collected and analyzed in terms of their correlation with microglia/macrophages.

Results: Microglia/macrophages constituted the major portions of the granulomas surrounding the eggs in both all human cases and S. japonicum egg-injected mice. Granuloma persisted in all patients accompanied by unremitted neurological symptoms, while in mice granuloma formation initiated on day 3, peaked on day 7 and subsided on day 30 post injection with S. japonicum eggs. No neurological abnormalities were observed in egg-injected mice except for significant weight decrease on day 3 compared with saline-injected control. M1 polarization of microglia/macrophages was confirmed in egg-injected mice 3 days post injection and in all human cases. M2 polarization was absent in human patients despite the duration of complaints but dominated in the whole progression of egg-induced pathology in mice until the elimination of eggs and subsidence of neuroinflammation on day 30 post injection.

Conclusions: Microglia/macrophages participated actively in the granuloma microenvironment of encephalic schistosomiasis japonicum in both human and mice. The polarization pattern of microglia/macrophages coincided with the symptomatic features in human cases and S. japonicum egg-injected mice, indicating M2 instead of M1 activation as a probably more important mediator in the battle against egg-induced pathology and concomitant manifestations. These new findings will shed light on the pathogenesis of NS from a brand-new perspective, and may contribute to the immunotherapy development for such disease, favoring perhaps M2 polarization of microglia/macrophages as a feasible strategy.
\end{abstract}

Keywords: Encephalic schistosomiasis japonicum, Granuloma microenvironment, Microglia/macrophages, Polarization

\footnotetext{
* Correspondence: hufeng@tjh.tjmu.edu.cn; tlei@tjh.tjmu.edu.cn

${ }^{1}$ Sino-German Neuro-Oncology Molecular Laboratory, Department of

Neurosurgery, Tongji Hospital, Tongji Medical College, Huazhong University

of Science and Technology, Wuhan, China

Full list of author information is available at the end of the article
}

(c) The Author(s). 2019 Open Access This article is distributed under the terms of the Creative Commons Attribution 4.0 International License (http://creativecommons.org/licenses/by/4.0/), which permits unrestricted use, distribution, and reproduction in any medium, provided you give appropriate credit to the original author(s) and the source, provide a link to the Creative Commons license, and indicate if changes were made. The Creative Commons Public Domain Dedication waiver (http://creativecommons.org/publicdomain/zero/1.0/) applies to the data made available in this article, unless otherwise stated. 


\section{Background}

Schistosomiasis is a prevalent parasitic disease which plagues more than 240 million people in 76 countries in the world [1]. Neuroschistosomiasis (NS), referring to schistosomal involvement of the CNS (central nervous system), is a severe form of presentation of schistosomiasis [2]. Schistosoma japonicum (S. japonicum), prevalent in Southeast Asia, mainly affects the brain (encephalic schistosomiasis japonicum), which constitutes $2-4 \%$ of all such cases [3, 4]. Although the pathogenesis of NS has not been fully elucidated, it is proposed that the deposition of parasite eggs in the nervous tissue induces an inflammatory response leading to the formation of granulomas, which is the basis of CNS-related clinical symptoms [2-5]. Common manifestations in CNS-infected patients include headache, vomiting, dizziness, convulsion and paralysis, adding to the global burden of schistosomiasis [6]. Despite the great progress in controlling the transmission of Schistosoma japonicum in China, there remains little known about the basic mechanisms underlying the pathophysiology of CNS infection [7].

Microglia/macrophages are the major immune cells involved in detection and subsequent elimination of pathogens and injured tissue in CNS [8]. However, very little is known about their roles in the granuloma formation surrounding the eggs and clinical significance in CNS. In order to clarify the pathological involvement of microglia/ macrophages in the pathogenesis of NS, as well as microglia/macrophages correlation with clinical features, staining techniques were applied to the granuloma tissues excised from 4 patients, obtained during neurosurgical operation in present study.

There are animal models of schistosomiasis presenting CNS infection. However, CNS involvement was very rare in natural infection progression, which contributed to the difficulty of research on it [9-11]. Microinjection of viable Schistosoma eggs into specific organs to establish schistosomiasis animal models has been reported to be a useful way in studying site-specific infections of Schistosoma [12-15]. Herein we apply microinjection technique in establishing encephalic schistosomiasis japonicum mice model to investigate the role of microglia/macrophages in its pathogenesis.

\section{Methods}

\section{Clinical data}

The study was conducted on tissues obtained from 4 cases of encephalic schistosomiasis japonicum. Associated clinical data was collected from archival files dating from 2013 to 2017 in the Department of Neurosurgery, Tongji Hospital, Tongji Medical College, Huazhong University of Science and Technology. The clinical features of these cases are summarized in Table 1.

\section{S. japonicum egg isolation}

S. japonicum-infected rabbits were generously provided by Dr. Fei Guan and Dr. Junli Xiao at the Department of Parasitology, Tongji Medical College, Huazhong University of Science and Technology. The rabbits were subcutaneously sedated with a ketamine/xylazine mix solution $(50 \mathrm{mg} / \mathrm{kg}$ and $3 \mathrm{mg} / \mathrm{kg}$, respectively) followed by intra-cardiac injection of sodium pentobarbital 6 weeks post-infection. The livers were minced, homogenized in a blender, resuspended with $1.2 \% \mathrm{NaCl}$ solution containing antibiotic-antimycotic solution (100 units Penicillin, $100 \mu \mathrm{g} / \mathrm{mL}$ Streptomycin and $0.25 \mu \mathrm{g} / \mathrm{mL}$ Amphotericin B, Sigma-Aldrich) and $0.25 \%$ trypsin, passed through a series of sieves with sequentially decreasing pore sizes $(500 \mu \mathrm{m}, 200 \mu \mathrm{m}$, and $100 \mu \mathrm{m})$, and finally retained on a $45 \mu \mathrm{m}$ sieve. The eggs were repeatedly washed to remove impurities and resuspended in $4{ }^{\circ} \mathrm{C} 0.9 \% \mathrm{NaCl}$ solution for injection. Control group was applied with equal volume of $0.9 \% \mathrm{NaCl}$.

\section{Mice model establishment}

Six to seven week-old female C57BL/6 mice were purchased from Animal Center of Tongji Hospital. Forty mice were randomly assigned into Egg and control groups. Animals were anesthetized with isoflurane and placed in a stereotactic frame. The skin was incised and one hole was drilled in the skull. Freshedly prepared S. japonicum eggs (500 eggs in $2 \mu \mathrm{l}$ of $0.9 \% \mathrm{NaCl}$ ) or saline was injected into the cortex. Each injection lasted $4 \mathrm{~min}$ with the needle left in situ for another $2 \mathrm{~min}$, then raised $0.5 \mathrm{~mm}$ and left another minute, before being slowly withdrawn. The incisions were closed with suture and treated once with topical antibiotic ointment. Neurological symptoms and weight was closely recorded after surgery. Mice were euthanized by $\mathrm{CO}_{2}$ at serial time points 3 to 30 days after brain injection, and brains processed for further histological analysis. All experimental procedures were carried out in accordance with the Institutional Animal Care and Use Committee guidelines and approved by Ethical Committee of Tongji Hospital, Tongji Medical College, Huazhong University of Science and Technlogy. Full exertions were made to lessen animal suffering.

\section{Histopathology, immunohistochemistry and immunofluorescence}

The tissues were routinely fixed in $10 \%$ buffered formalin for at least $12 \mathrm{~h}$, and processed and embedded in paraffin blocks; $5 \mu \mathrm{m}$ paraffin sections were microtomed, gradually dewaxed into the water, and HE staining was performed. The histopathological features were evaluated under light microscope (Nikon 80i).

For immunohistochemistry, the paraffin sections were gradually dewaxed into the water, then immersed in 0.01 $\mathrm{mol} / \mathrm{L}$ citrate buffers and microwaved for antigen retrieval. 
Table 1 Summary of clinical characteristics of encephalic schistosomiasis japonicum

\begin{tabular}{|c|c|c|c|c|c|}
\hline Case No. & Sex & Presenting symptoms & Site & Therapy performed & Follow-up \\
\hline 1 & M & $\begin{array}{l}\text { Headache with vomiting } \\
\text { for } 9 \text { days }\end{array}$ & Single nodule in right cerebellum & Total surgical resection & $\begin{array}{l}\text { Well-controlled } 4 \text { years with } \\
\text { continuous follow-up }\end{array}$ \\
\hline 2 & $\mathrm{~F}$ & Epilepsy for 3 days & Single nodule in left parietal lobe & Total surgical resection & $\begin{array}{l}\text { Seizure-free } 2 \text { years, loss of } \\
\text { contact afterwards }\end{array}$ \\
\hline 3 & M & $\begin{array}{l}\text { Headache and motor } \\
\text { aphasia for } 20 \text { days }\end{array}$ & $\begin{array}{l}\text { Multiple nodules in left frontotemporal } \\
\text { lobe }\end{array}$ & Total surgical resection & Loss of contact \\
\hline 4 & M & Epilepsy for 1 week & $\begin{array}{l}\text { Multiple nodules in left frontal, parietal } \\
\text { and temporal lobes }\end{array}$ & Total surgical resection & $\begin{array}{l}\text { Seizure-free } 2 \text { years with continuous } \\
\text { follow-up }\end{array}$ \\
\hline
\end{tabular}

After incubation of primary antibodies on the sections according to the protocol from the suppliers, the standard DAKO ChemMate ${ }^{\mathrm{TM}}$ EnVision Kit (horseradish peroxidase (HRP)/3,3' -diaminobenzidine (DAB), rabbit/mouse) (Shanghai Gene Company, Shanghai, China), based on the two-step labeled HRP method, were used according to the manufacturer's instructions. PBS solution instead of the primary antibody was used as the negative control during immunohistochemical staining. DAB was used as the chromonogen. The deposition of granular dark brown pigments were considered as positive staining.

For immunofluorescence, the paraffin sections were gradually dewaxed into the water, then immersed in $0.01 \mathrm{~mol} / \mathrm{L}$ citrate buffers and microwaved for antigen retrieval. After incubation of primary antibodies on the sections according to the protocol from the suppliers, the secondary antibodies labelled with specific fluorescent compounds were used. PBS solution instead of the primary antibody was used as the negative control. DAPI was used as the chromonogen. Images were captured using fluorescence microscope (Leica AF 6000).

The primary antibodies used in this study are summarized in Table 2.

\section{Statistical analysis}

Unpaired t test with Welch's correction was used to compare the results between control and egg-injected groups, and data were expressed as mean \pm standard deviation. $\mathrm{P}<0.05$ was considered statistically significant.

\section{Results}

\section{Clinical features}

The age of the patients in this group ranged from 27 to 51 years (median age of 47.25 years). The male to female ratio was 3:1. Every patient had a history of contact with schistosome-infected water. None of the patients presented

Table 2 Primary antibodies used in this study

\begin{tabular}{lll}
\hline Primary antibody & Dilution & Company \\
\hline IBA1 ab5076 & $1: 500$ & Abcam \\
iNOS ab178945 & $1: 500$ & Abcam \\
Arg1 ab212522 & $1: 500$ & Abcam \\
\hline
\end{tabular}

with gastrointestinal manifestations such as hepatosplenomegaly, ascites and esophagogastric varices. In contrast, all of the patients presented with headache and two of them presented with seizures, with one presenting with motor aphasia. The duration of their complaints ranged from 3 to 20 days. All the patients received anti-schistosomal medical treatment but remission was not achieved and thus surgery was performed.

\section{Preoperative neuroradiology}

MRI scans of the patients revealed either single or multiple nodular schistosomal granulomas (Fig. 1). Depending upon the patient, the nodules were located uniquely or in combination (multiple) in one side of the parietal, frontal and/or temporal lobes and/or cerebellum. For example, in case 2 (Fig. 1a-d), the lesions were located only in the left parietal lobe. In contrast, in case 4 (Fig. 1e-h), multiple lesions were found in the left frontal, parietal and temporal lobes. T2WI (T2-weighted image) showed edema of differing extents surrounding the nodules. Enhanced scanning indicated enhancement of the nodules.

\section{Pathological features of brain tissue from neuroschistosomiasis patients}

Microscopically, presence of parasite eggs was clearly observed in all cases (Fig. 2). Histological staining demonstrated that the observable eggs were surrounded by multinucleated giant cells indicating an immune response forming granulomas.

\section{Assessment of microglia /macrophage abundance in the brain tissue from neuroschistosomiasis patients}

In all 4 cases, the granulomas appeared to be accumulated by microglia/macrophages surrounding the eggs as indicated by large amounts of dark brown staining using an antibody against the specific IBA1 (Ionized calcium binding adapter molecule 1) marker [16] for such cells (Fig. 3a-c). Absorption control staining was carried out to further confirm the specificity of the antibody (Additional file 1: Figure S1). 

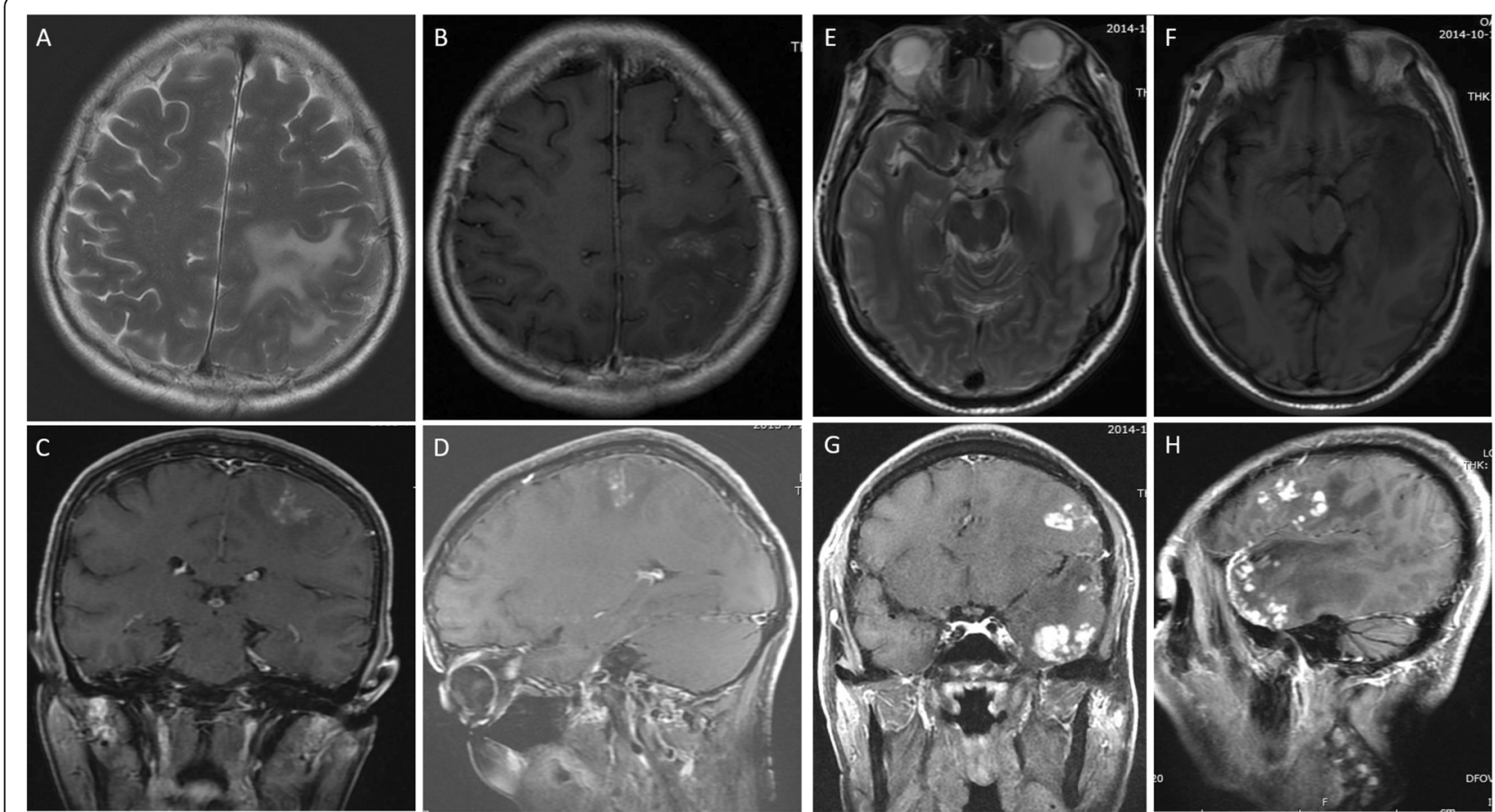

Fig. $1 \mathrm{MRl}$ features of the representative encephalic Schistosomiasis japonicum cases (a-d from Case 2, e-h from Case 4). a: T2 weighted (W) image showed a single-nodular lesion located in the left parietal lobe with perilesional edema by axial scanning. b-d: T1 W image after gadolinium injection showed single-nodular enhancement with perilesional edema by axial (b), coronal (c), and sagittal scanning (d). e: T2 W image showed edema of left temporal lobe by axial scanning. f: T1 W image showed the involvement of the left temporal lobe in multi-nodular lesions by axial scanning. $\mathbf{g}, \mathbf{h}$ : T1 W image showed multiple-nodular schistosomal lesions located in frontal, parietal and temporal lobes with enhancement of the nodules

\section{Polarization of microglia/macrophages in CNS} Schistosomiasis patients

The polarization of the microglia/macrophages was determined using specific antibodies for M1 marker (iNOS, inducible nitric oxide synthase) and M2 marker (Arginase1, Arg1) and merging the images with that given by IBA-1 (Fig. 3d-h). Interestingly, in all cases the polarization of the microglia/macrophages was of the M1 type, the staining being negative for Arg1. The merged image demonstrated that the same cells were positively stained for both iNOS and IBA-1 (Fig. 3d-f).

\section{Neurological abnormalities of mice model}

Although no neurological symptoms like seizure and hemiplegia were observed in either of the groups after recovery from anesthesia, the weight of both groups decreased significantly in 3 days post-operation $(\mathrm{P}<0.001)$ (Fig. 4), and increased steadily after that. More interestingly, there was

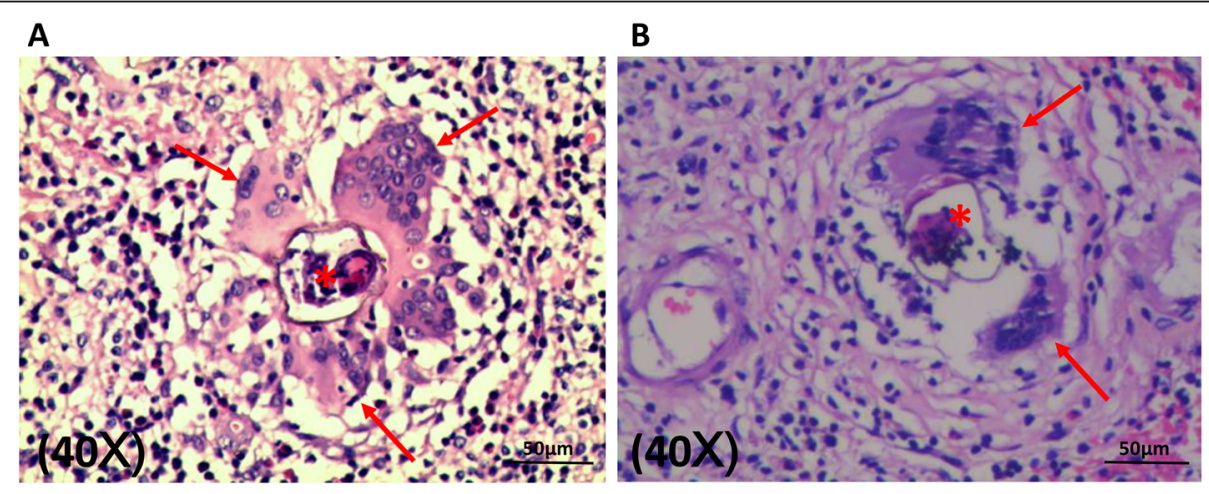

Fig. 2 Pathological features of brain tissue from neuroschistosomiasis patients (a from Case 1, b from Case 2). Schistosomal eggs were surrounded by multinucleated giant cells. Asterisk = S. japonicum egg, Arrow = multinucleated giant cells 

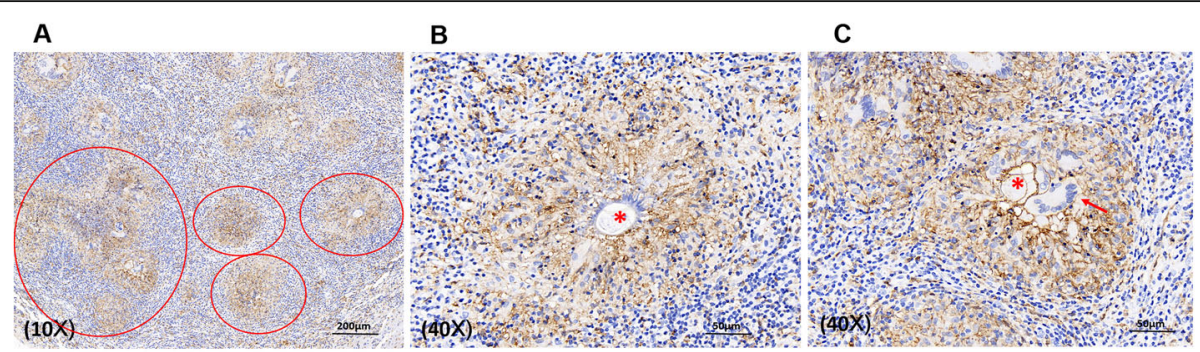

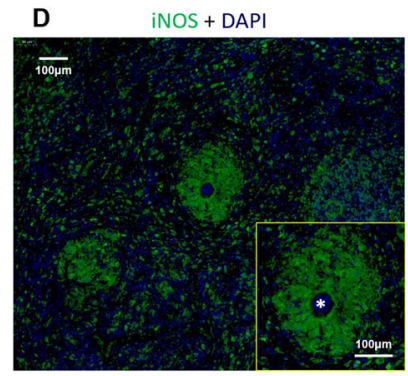

G

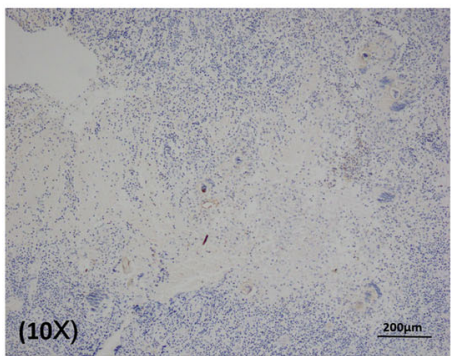

IBA1 + DAPI

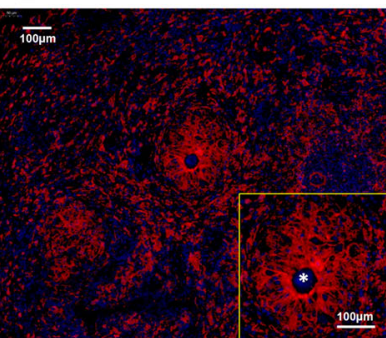

F iNOS+IBA1+DAPI

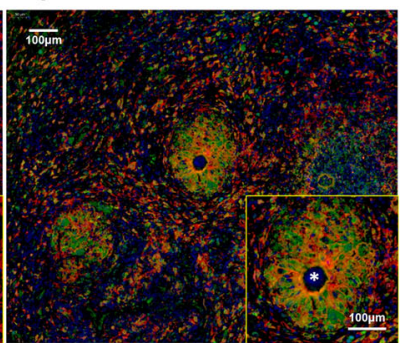

H

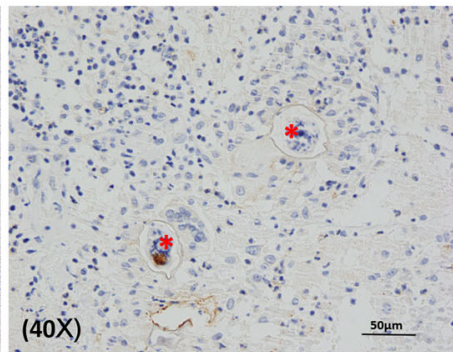

Fig. 3 Identification and polarization of microglia/macrophages in the granuloma microenvironment of neuroschistosomiasis patients. a-c: Microglia/macrophages constituted the major portions of the granulomas (circle) surrounding the eggs as highlighted by IBA1 staining. The schistosomal egg shell (asterisk) can be observed clearly with multinucleated giant cells (arrow) in the vicinity. d-f: Immunofluorescent staining indicating M1 polarization of microglia/macrophages. iNOS (green) and IBA1 (red) were well co-expressed in the vicinity of the eggs (asterisk). Merged image demonstrated that the same cells were exactly the M1 type microglia/macrophages. $\mathbf{g}$-h: Immunohistochemical staining illustrating negative expression of M2 marker (Arg1) of microglia/macrophages surrounding the eggs (asterisk)

significant difference in the weight of two groups 3 days post-operation with egg group decreasing in a greater degree $(\mathrm{P}<0.001)$ (Fig. 4).

\section{S. japonicum egg injection-induced pathological process in mice}

As evidenced by results from encephalic schistosomiasis japonicum patients, pathogenesis and concomitant morbidity is the result of S. japonicum egg deposition in cerebral tissue. However, the whole pathological process of cerebral infection has never been reported and thus hindered its further investigation. To address this issue, we directly microinjected freshly-prepared viable $S$. japonicum eggs into the cerebral cortex of female C57BL/6 mice. The initial injection site response resolved entirely by day 3 in mice injected with egg-free control vehicle, leaving puncture path visible (Fig. 5a-d). Egg-injected group demonstrated inflammatory infiltrate and granuloma formation surrounding S. japonicum eggs as early as day 3
(Fig. 5e and i). Inflammation response and granuloma continued to progress and peaked on day 7 (Fig. $5 f$ and j). Interestingly, unlike findings in humans, S. japonicum egg disintegration was observed on day 14 and inflammation response diminished greatly (Fig. $5 \mathrm{~g}$ and $\mathrm{k}$ ). On day 30 the inflammation subsided completely and $S$. japonicum eggs vanished without trace (Fig. 5h) except for the ones which we think were unviable and unable to secrete soluble antigen to induce immune response (Fig. $5 \mathrm{l}$ ).

\section{Microglia /macrophage accumulation in mice model}

In accordance with results in encephalic schistosomiasis japonicum patients, granulomas appeared to be mostly composed of microglia/macrophages surrounding the eggs as confirmed by immunohistochemistry for IBA1 on day 3 and day 7 (Fig. 6bc), while control exhibited neither granuloma formation nor microglia/macrophages aggregation (Fig. 6a). With the decomposition of S. japonicum eggs on day 14, granuloma shrank and microglia/macrophages 


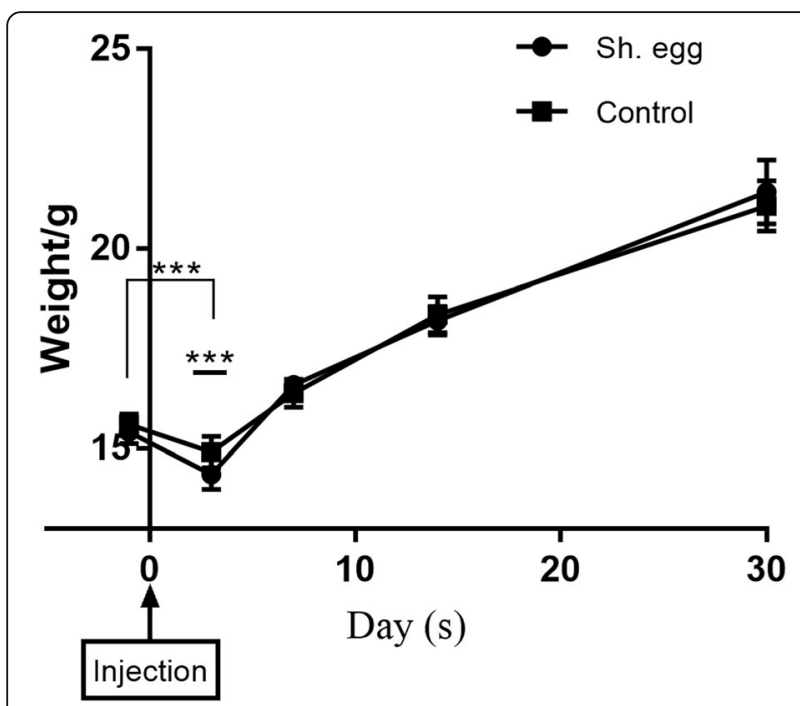

Fig. 4 Weight of egg-injected and control groups over time. Unpaired t test with Welch's correction was used to compare the weight between the two groups, and data were expressed as mean \pm standard deviation. $\mathrm{P}<0.05$ was considered statistically significant. The data showed significant decrease of the weight on day 3 in both groups compared to day $\left.0{ }^{* * *} P<0.001\right)$ and significant difference in the weight of two groups on day $\left.3{ }^{* * *} P<0.001\right)$ abundance decreased significantly (Fig. 6d). S. japonicum eggs were always closely surrounded by microglia/macrophages (Fig. 6e-g). During egg elimination process, microglia/macrophages could even invade inside the eggs (Fig. 6f). These findings added to the fact that microglia/ macrophages participated actively in the S. japonicum egginduced immune response and played a primary role in the elimination of S. japonicum eggs.

\section{Polarization of microglia/macrophages in S. japonicum egg-induced granulomas in mice}

M1 polarization of microglia/macrophages existed in $S$. japonicum egg-induced granulomas only on day 3 post injection (Fig. 7a) and specific marker iNOS staining was negative after that (Fig. 7b-d), while M2 polarization sustained from day 3 until inflammation subsidence on day 30 , as indicated by positive expression of both Arg1 (red) and IBA1 (green), colocalization of which confirmed M2 microglia/macrophages in the vicinity of the eggs (Fig. 7e-m).

\section{Discussion}

Schistosomiasis japonicum is an important disease to investigate since it is of prevalence in Asian countries and can infect workers such as rice farmers and fishermen, who are an integral part of the food economy in these

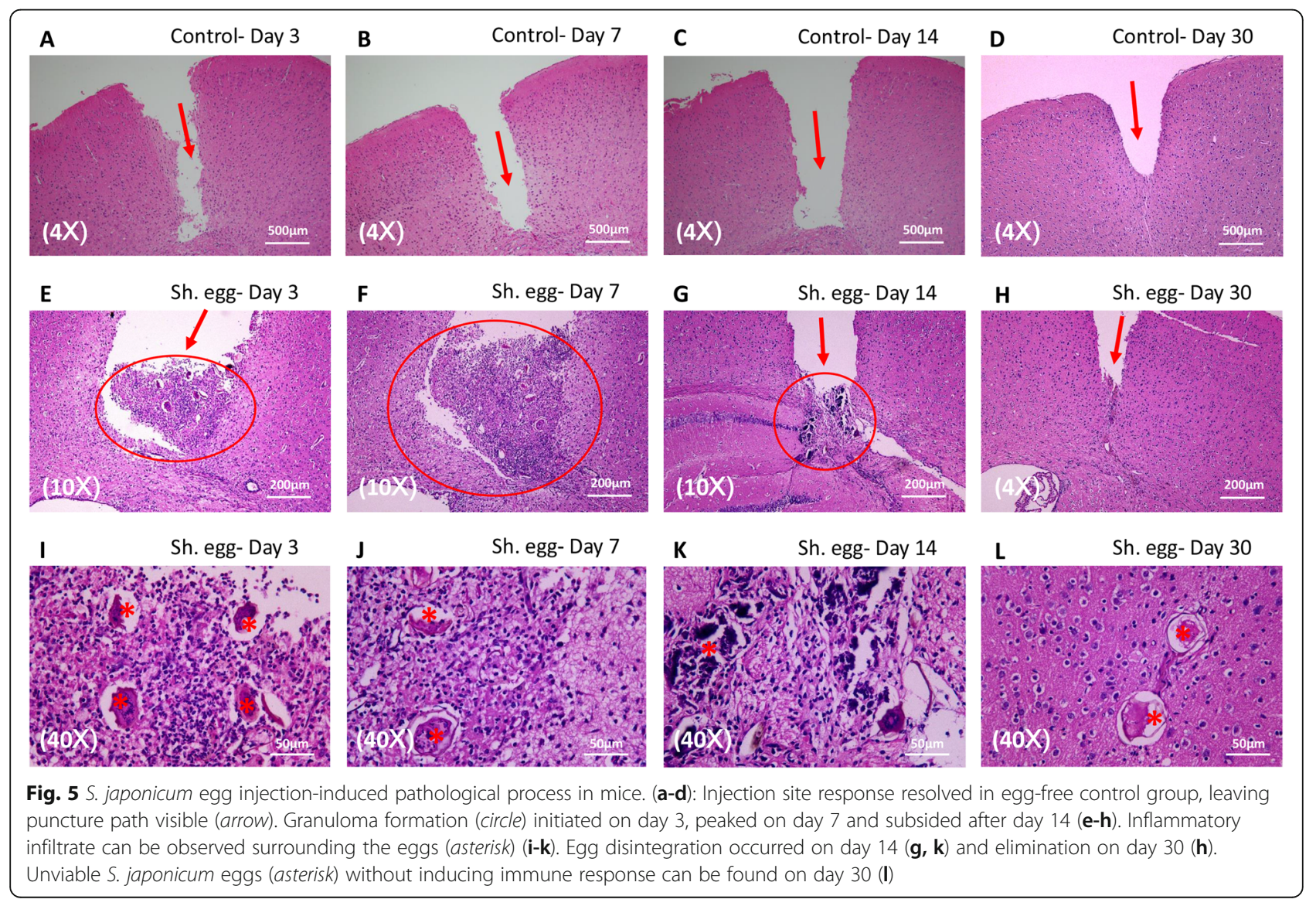




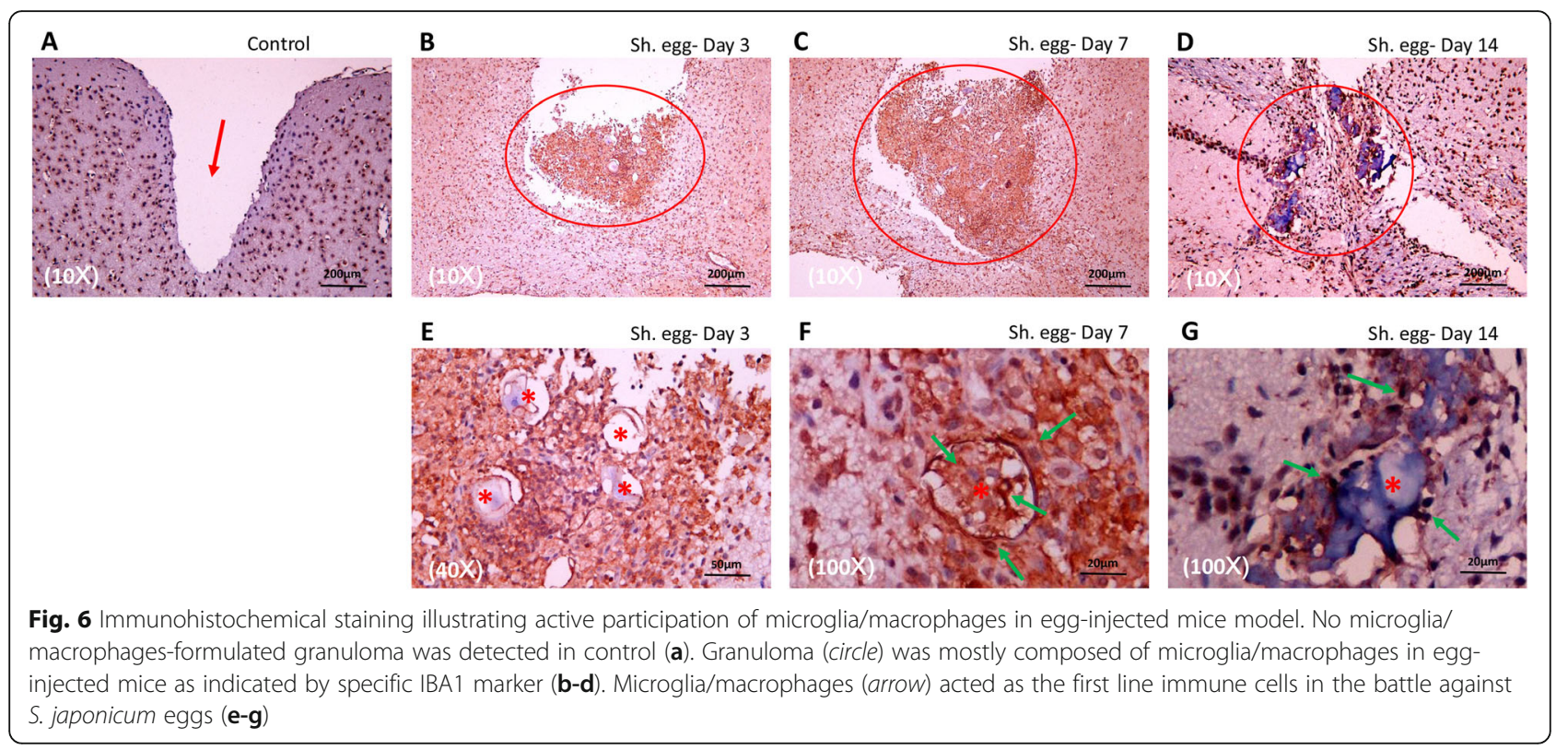

countries [17]. The involvement of the CNS, which is relatively rare, can cause a series of the most harzardous symptoms such as epilepsy, hemiplegia and intracranial hypertension contributing to the medical burden $[3,4]$. It is surprising, therefore, that there is a paucity of clinical and scientific investigations of this disease in the literature, although the life cycle of the parasite is quite well established [17]. It is hypothesized by some researchers that the eggs can invade the CNS from the portal mesenteric and pelvic system through two routes: Batson's venous plexus and the arterial system $[2,18]$. The trapped eggs can induce a host's immune response and cause the formation of granulomas whose mass effect and intrinsic immune properties are thought to be the basis of clinical symptoms [2-4, 18, 19]. Although there is a lack of study thoroughly demonstrating the microenvironment of the granuloma formation in encephalic Schistosomiasis japonicum, researches focusing on Schistosomiasis mansoni (another species of genus Schistosoma, mainly prevalent in Africa and Brazil) revealed the involvement of monocytes, lymphocytes and eosinophils along with soluble factors in CNS infection [5]. Microglia/macrophages are among the first effectors to pathogen invasion, responding via releasing of various cytokines and clearance of pathogens and debris [20, 21]. However, none of the investigation mentioned the role of microglia/macrophages in the granuloma microenvironment in neuroschistosomiasis. Therefore, we undertook this present study in order to gain a better understanding of the disease process with the emphasis on the role of microglia/macrophages in such disease. The findings demonstrate that the lesions can occur either at single or multiple sites in the brain and lead to typical immune type histological changes in patients, where multinucleated giant cells, which is thought to be the result of fusion of microglia/macrophages, [22] can be found to be located closely to the eggs. In addition, our series of patients as well as mice model showed, for the first time, that microglia/macrophages constituted the major portions of the granulomas surrounding the eggs, demonstrating their important role in this disease. In egg-injected mice, microglia/macrophages could invade inside the eggs on day 7 post injection, followed by the decomposition and elimination of eggs on day 14, revealing their active participation in mediating local pathogen control.

During the course of infection, the symptomatic and pathological features of mice model were different from those of CNS-infected patients in many aspects. The inflammation and granuloma formation in mice model existed in early stage $(<14 \mathrm{~d})$, which resembled findings in patients as revealed by both neuroradiology and microscopy, and subsided completely on day 30 . However, inflammation and granuloma persisted without signs of remission even when anti-schistosomal medical treatment was applied in all the patients. More interestingly, the injected eggs gradually lost their original shape over the course, disintegrated and disappeared in the end, while in all human cases eggs persisted despite the duration of their complaints and anti-schistosomal medical treatment. Moreover, no neurological symptom was observed in mice model in contrast to 4 cases who exhibited severe complications such as headache and seizure before neurosurgery. In parallel, reasonably, the weight decrease of control mice on day 3 might be attributed to surgical procedure and confounding factors, while the 

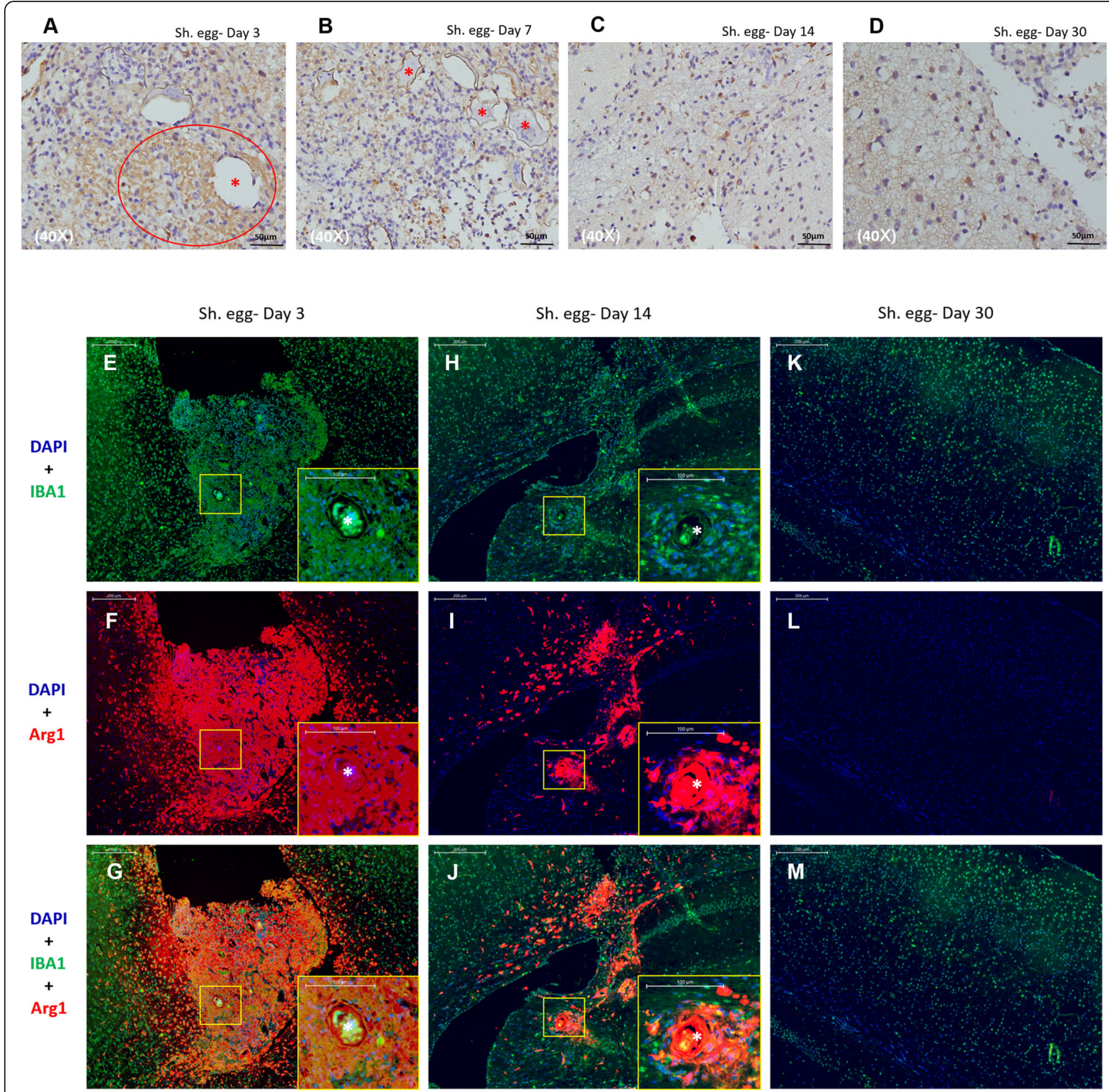

Fig. 7 Polarization of microglia/macrophages in S. japonicum egg-induced granulomas in mice. a-d: Immunohistochemical staining illustrating M1 polarization of microglia/macrophages on day 3 in egg-injected mice as indicated by positive iNOS expression (circle) surrounding the eggs (asterisk). e-m: Immunofluorescent staining indicating M2 polarization of microglia/macrophages in egg-injected mice. M2 polarization sustained from day 3 until inflammation subsidence on day 30, as indicated by positive expression of both Arg1 (red) and IBA1 (green). S. japonicum eggs: Asterisk

weight difference between control and egg group at the same time might be due to egg-induced immune response.

Given the importance of microglia/macrophages in the granuloma microenvironment of encephalic schistosomiasis japonicum in both human and mice as evidenced above, we further studied their polarization pattern in attempt to explain the discordance between patients and mice model.
As an important fighter against pathogenic infections in CNS, resting microglia may undertake two activation pathways: the classical known as M1, or the alternative known as M2 [23, 24]. Macrophages, peripheral counterparts of microglia, can infiltrate the brain and contribute to the homeostasis of it together with resident microglia, both of which share similar biological functions and polarization patterns $[25,26]$. M1 polarization of microglia/macrophages is thought to be involved in the initiation and 
perpetuation of neuroinflammation, whereas $\mathrm{M} 2$ reaction is more likely related to the remission of neuroinflammation through elimination of pathogens and damaged tissues [8]. The nitric oxide, produced by iNOS (an M1 marker), plays a key role in inhibiting the growth of Schistosoma japonicum in rats [27]. It is also well known that M1 microglia/ macrophage-related neuroinflammation can lead to secondary brain defects after some conditions such as stroke and traumatic brain injury [28, 29]. M1 phenotype may also create an unfavorable microenvironment for CNS recovery by secreting destructive factors that aggravate neurological injury [30-32]. By contrast, M2 microglia/macrophages can not only resolve neuroinflammation by clearing cellular debris and pathogens but can also promote restorative processes by releasing numerous protective and trophic factors [33-35]. Arginase1, among the first discovered M2 markers, can down-regulate nitric oxide synthesis and initiate tissue regeneration and fibrosis [36]. All the human cases in our present study exhibited a microglial/macrophage mediated response perhaps involving only an M1 polarization (indicated by iNOS) at the time after resection, while in egg-injected mice M1 phenotype existed on day 3 and M2 activation (indicated by arginase1) persisted throughout the immune response until neuroinflammaton subsided on day 30 . These findings in combination with the manifestations in human cases and mice model demonstrated a strong possibility that it was the M1 polarization of microglia/macrophage that mediated the persistent neuroinflammation, which further harmed the brain and induced a series of additional symptoms in human cases and weight decrease in egg-injected mice model. M2 activation, unobserved in human patients while dominating in egg-injected mice model, might be the dictator of $S$. japonicum elimination, neuroinflammation remission and absence of symptoms in mice. However, due to the limitation of human samples, all the patients have been treated with different medications such as anti-schistosomiasis drug, steroid or mannitol which may affect the phenotype of microglia in the brain. More patient samples are needed for further identifying the phenotype of microglia/macrophages at different stages of neurochistosomiasis.

The mechanism of microglia/macrophage polarization in CNS-infected schistosomiasis has never been reported. Soluble egg antigen secreted by viable $S$. japonicum eggs can activate M2 macrophages in mice via the STAT6 and PI3K Pathways in vitro [37]. Numerous factors existing in the microenvironment of CNS can polarize microglia/macrophage to different phenotypes [38]. IFN- $\gamma$, TNF and IL-2 secreted by type $1 \mathrm{~T}$-helper cells can induces M1 polarization while IL-4 secreted by type $2 \mathrm{~T}$-helper cells and regulatory T cells can cause M1 activation [39, 40]. Soluble factors released from injured neurons and extracellular matrix proteins can induce M1 and M2 polarization respectively $[29,41]$. Although the mechanism of microglia/ macrophage polarization still needs to be unveiled in neuroschistosomiasis, our present study revealed M2 instead of M1 as a neuroinflammation suppressor and symptom controller in encephalic schistosomiasis japonicum, which will shed light on the possible immunotherapy strategies in the control of such disease.

\section{Conclusions}

This report is the first to thoroughly investigate the clinical features and microglia/macrophages features of both patients and mice model with specific CNS-related Schistosoma japonicum infection. We first proved the majority part of the granulomas surrounding the eggs to be composed of microglia/macrophages in human and mice model by staining technique. The polarization pattern of microglia/macrophages was then examined and the manifestations of both patients and mice model were recorded at the same time. M1- dominant polarization in patients and M2-dominant activation in egg-injected mice coincided with the complaints in patients and symptomlessness in mice model, revealing that microglia/macrophages acted as double-edged swords in the battle of $S$. japonicum egg-induced pathology. These new findings will shed light on the pathogenesis of NS from a brand-new perspective, and may contribute to the immunotherapy development for such disease, favoring perhaps M2 polarization of microglia/macrophages as a feasible strategy.

\section{Supplementary information}

Supplementary information accompanies this paper at https://doi.org/10. 1186/s12879-019-4725-5.

Additional file 1: Figure S1. Absorption control staining using human Iba1 peptide (Abcam, ab23067) confirming the specificity of Iba1 antibody (Abcam, ab5076). The peptide to antibody mixture was made at a working dilution of 10:1 (molar ratio) and pre-incubated overnight at $4{ }^{\circ} \mathrm{C}$. The preabsorbed antibody was then incubated with tissue excised from neuroschistosomiasis patients in place of the primary antibody alone. Iba1 staining surrounding the eggs (asterisk) was abolished after the antibody was first preabsorbed with Iba1 peptide (A, B) compared with Fig. 3a-c.

\section{Abbreviations \\ Arg1: Arginase1; CNS: Central nervous system; IBA1: Ionized calcium binding adapter molecule 1; iNOS: Inducible nitric oxide synthase; \\ NS: Neuroschistosomiasis}

\section{Acknowledgements}

We would like to thank Dr. Eric Adams for reading and correcting the English in this manuscript.

We also thank Dr. Fei Guan and Dr. Junli Xiao for their technical assistance in the isolation of S. japonicum eggs.

\section{Authors' contributions}

$\mathrm{TL}$ and $\mathrm{FH}$ designed and supervised the study and revised the manuscript. $\mathrm{ZT}$ and $\mathrm{ZL}$ conducted the experiments and wrote the manuscript. $\mathrm{HZ}$ and $\mathrm{KS}$ evaluated the clinical data and provided the clinical samples. ZT, ZL and ZZ analyzed the data. All authors read and approved the manuscript. 


\section{Funding}

This work received financial support from National Natural Science Foundation of China (grant No. 81602202) and Hubei Province health and family planning scientific research project (grant No. Wj2017X008). The funders had no role in study design, the decision to publish, or preparation of the manuscript.

\section{Availability of data and materials}

The datasets used and/or analyzed in the current study were available from the corresponding author on reasonable request.

\section{Ethics approval and consent to participate}

Animal experiments in this study were implemented according to the Institutional Animal Care and Use Committee guidelines and approved by Ethical Committee of Tongji Hospital, Tongji Medical College, Huazhong University of Science and Technlogy, Wuhan, China. Full exertions were made to lessen animal suffering and to minimize the number of experimental animals. Written informed consent of the patients was obtained.

\section{Consent for publication}

Not applicable.

\section{Competing interests}

The authors declare that they have no competing interests.

\section{Author details}

${ }^{1}$ Sino-German Neuro-Oncology Molecular Laboratory, Department of Neurosurgery, Tongji Hospital, Tongji Medical College, Huazhong University of Science and Technology, Wuhan, China. ${ }^{2}$ Department of Orthopedics, Tongji Hospital, Tongji Medical College, Huazhong University of Science and Technology, Wuhan, China. ${ }^{3}$ Present Address: Department of Neurosurgery, Philipps University Marburg, Baldingerstr, Marburg, Germany.

\section{Received: 19 June 2019 Accepted: 22 December 2019}

\section{Published online: 30 December 2019}

\section{References}

1. Lo NC, Gurarie D, Yoon N, Coulibaly JT, Bendavid E, Andrews JR, et al. Impact and cost-effectiveness of snail control to achieve disease control targets for schistosomiasis. Proc Natl Acad Sci U S A. 2018;115(4):E584E91.

2. Ferrari TC, Moreira PR. Neuroschistosomiasis: clinical symptoms and pathogenesis. Lancet Neurol. 2011;10(9):853-64.

3. Lei T, Shu K, Chen X, Li L. Surgical treatment of epilepsy with chronic cerebral granuloma caused by Schistosoma japonicum. Epilepsia. 2008;49(1):73-9.

4. Shu K, Zhang S, Han L, Lei T. Surgical treatment of cerebellar schistosomiasis. Neurosurgery. 2009;64(5):941-3 discussion 3-4.

5. Ferrari TC, Gazzinelli G, Correa-Oliveira R. Immune response and pathogenesis of neuroschistosomiasis mansoni. Acta Trop. 2008:108(2-3):83-8.

6. Sokolow $\mathrm{SH}$, Wood $\mathrm{CL}$, Jones IJ, Lafferty KD, Kuris AM, Hsieh MH, et al. To reduce the global burden of human Schistosomiasis, use 'Old Fashioned' snail control. Trends Parasitol. 2018;34(1):23-40.

7. Qian C, Zhang Y, Zhang X, Yuan C, Gao Z, Yuan H, et al. Effectiveness of the new integrated strategy to control the transmission of Schistosoma japonicum in China: a systematic review and meta-analysis. Parasite. 2018;25:54

8. Kettenmann H, Hanisch UK, Noda M, Verkhratsky A. Physiology of microglia. Physiol Rev. 2011;91(2):461-553.

9. Lambertucci JR, Fidelis TA, Pereira TA, Coelho PM, Araujo N, Souza MM, et al. Brain schistosomiasis in mice experimentally infected with Schistosoma mansoni. Rev Soc Bras Med Trop. 2014:47(2):251-3.

10. Silva LM, Oliveira CN, Andrade ZA. Experimental neuroschistosomiasis: inadequacy of the murine model. Memorias do Instituto Oswaldo Cruz. 2002;97(4):599-600.

11. Aloe L, Moroni R, Fiore M, Angelucci F. Chronic parasite infection in mice induces brain granulomas and differentially alters brain nerve growth factor levels and thermal responses in paws. Acta Neuropathol. 1996:92(3):300-5.

12. de Carvalho TPV, Ferrari TCA, de Santana JM, Viana VAS, Santos JAC. Do Nascimento WC, et al. development of an experimental model of schistosomal myeloradiculopathy. Acta Trop. 2017;167:142-7.
13. Wang P, Wang D, Chen SJ, Wu MC, Cheng XL, Li JC, et al. Establishment of a cerebral schistosomiasis experimental model in rabbits. Neurosci Bull. 2011;27(2):91-8

14. Richardson ML, Fu CL, Pennington LF, Honeycutt JD, Odegaard Jl, Hsieh YJ, et al. A new mouse model for female genital schistosomiasis. PLoS Negl Trop Dis. 2014;8(5):e2825.

15. Fu CL, Odegaard Jl, Herbert DR, Hsieh MH. A novel mouse model of Schistosoma haematobium egg-induced immunopathology. PLoS Pathog. 2012;8(3):e1002605.

16. Ito D, Imai Y, Ohsawa K, Nakajima K, Fukuuchi Y, Kohsaka S. Microgliaspecific localisation of a novel calcium binding protein, Iba1. Brain Res Mol Brain Res. 1998;57(1):1-9.

17. Colley DG, Bustinduy AL, Secor WE, King CH. Human schistosomiasis. Lancet 2014;383(9936):2253-64

18. Pittella JE. Neuroschistosomiasis. Brain Pathol. 1997:7(1):649-62.

19. Palin MS, Mathew R, Towns G. Spinal neuroschistosomiasis. Br J Neurosurg. 2015:29(4):582-4.

20. Klein RS, Hunter CA. Protective and pathological immunity during central nervous system infections. Immunity. 2017:46(6):891-909.

21. Franco R, Fernandez-Suarez D. Alternatively activated microglia and macrophages in the central nervous system. Prog Neurobiol. 2015;131:65-86.

22. Beyer M, Gimsa U, Eyupoglu IY, Hailer NP, Nitsch R. Phagocytosis of neuronal or glial debris by microglial cells: upregulation of MHC class II expression and multinuclear giant cell formation in vitro. Glia. 2000;31(3):262-6.

23. Wolf SA, Boddeke HW, Kettenmann H. Microglia in physiology and disease. Annu Rev Physiol. 2017;79:619-43.

24. Yeh $\mathrm{H}$, Ikezu T. Transcriptional and epigenetic regulation of microglia in health and disease Trends Mol Med. 2019:25(2):96-111.

25. Hu X, Leak RK, Shi Y, Suenaga J, Gao Y, Zheng P, et al. Microglial and macrophage polarization-new prospects for brain repair. Nat Rev Neurol. 2015;11(1):56-64.

26. Prinz M, Priller J. Microglia and brain macrophages in the molecular age: from origin to neuropsychiatric disease. Nat Rev Neurosci. 2014; 15(5):300-12.

27. Shen J, Lai DH, Wilson RA, Chen YF, Wang LF, Yu ZL, et al. Nitric oxide blocks the development of the human parasite Schistosoma japonicum. Proc Natl Acad Sci U S A. 2017;114(38):10214-9.

28. Kumar A, Alvarez-Croda DM, Stoica BA, Faden Al, Loane DJ. Microglial/ macrophage polarization dynamics following traumatic brain injury. J Neurotrauma. 2016:33(19):1732-50.

29. Hu X, Li P, Guo Y, Wang H, Leak RK, Chen S, et al. Microglia/macrophage polarization dynamics reveal novel mechanism of injury expansion after focal cerebral ischemia. Stroke. 2012;43(11):3063-70.

30. Ekdahl CT, Claasen JH, Bonde S, Kokaia Z, Lindvall O. Inflammation is detrimental for neurogenesis in adult brain. Proc Natl Acad Sci U S A. 2003; 100(23):13632-7.

31. Butovsky O, Ziv Y, Schwartz A, Landa G, Talpalar AE, Pluchino S, et al. Microglia activated by IL-4 or IFN-gamma differentially induce neurogenesis and oligodendrogenesis from adult stem/progenitor cells. Mol Cell Neurosci. 2006;31(1):149-60.

32. Roughton K, Andreasson U, Blomgren K, Kalm M. Lipopolysaccharideinduced inflammation aggravates irradiation-induced injury to the young mouse brain. Dev Neurosci. 2013:35(5):406-15.

33. Miron VE, Boyd A, Zhao JW, Yuen TJ, Ruckh JM, Shadrach JL, et al. M2 microglia and macrophages drive oligodendrocyte differentiation during CNS remyelination. Nat Neurosci. 2013;16(9):1211-8.

34. Mosser DM, Edwards JP. Exploring the full spectrum of macrophage activation. Nat Rev Immunol. 2008;8(12):958-69.

35. David S, Kroner A. Repertoire of microglial and macrophage responses after spinal cord injury. Nat Rev Neurosci. 2011;12(7):388-99.

36. Rath M, Muller I, Kropf P, Closs El, Munder M. Metabolism via Arginase or nitric oxide synthase: two competing arginine pathways in macrophages. Front Immunol. 2014;5:532.

37. Tang $H$, Liang YB, Chen ZB, Du LL, Zeng LJ, Wu JG, et al. Soluble egg antigen activates M2 macrophages via the STAT6 and PI3K pathways, and Schistosoma Japonicum alternatively activates macrophage polarization to improve the survival rate of septic mice. J Cell Biochem. 2017:118(12):4230-9.

38. Du L, Zhang Y, Chen Y, Zhu J, Yang Y, Zhang HL. Role of microglia in neurological disorders and their potentials as a therapeutic target. Mol Neurobiol. 2017;54(10):7567-84. 
39. Biswas SK, Mantovani A. Macrophage plasticity and interaction with lymphocyte subsets: cancer as a paradigm. Nat Immunol. 2010;11(10): 889-96.

40. Beers DR, Henkel JS, Zhao W, Wang J, Huang A, Wen S, et al. Endogenous regulatory $\mathrm{T}$ lymphocytes ameliorate amyotrophic lateral sclerosis in mice and correlate with disease progression in patients with amyotrophic lateral sclerosis. Brain. 2011;134(Pt 5):1293-314.

41. Rolls A, Shechter R, London A, Segev Y, Jacob-Hirsch J, Amariglio N, et al. Two faces of chondroitin sulfate proteoglycan in spinal cord repair: a role in microglia/macrophage activation. PLoS Med. 2008;5(8):e171.

\section{Publisher's Note}

Springer Nature remains neutral with regard to jurisdictional claims in published maps and institutional affiliations.

Ready to submit your research? Choose BMC and benefit from:

- fast, convenient online submission

- thorough peer review by experienced researchers in your field

- rapid publication on acceptance

- support for research data, including large and complex data types

- gold Open Access which fosters wider collaboration and increased citations

- maximum visibility for your research: over $100 \mathrm{M}$ website views per year

At BMC, research is always in progress.

Learn more biomedcentral.com/submissions 